\title{
Modelo Matemático apoiado por um Algoritmo Genético para classificação de Fake News na Web
}

\author{
Ana Luisa D. Almeida ${ }^{1}$, Gabriel C. Carrara ${ }^{1}$, Isabele B. Prates ${ }^{1}$, \\ Letícia C. T. Nascimento ${ }^{1}$, Pedro H. O. Souza ${ }^{1}$, Thiago R. Almeida ${ }^{1}$, \\ Renato C. Cani ${ }^{1}$, João Gabriel R. Silva ${ }^{1}$ \\ ${ }^{1}$ Instituto Federal de Mato Grosso - campus Pontes e Lacerda (IFMT) \\ Caixa Postal 99 - 78.250-000 - Pontes e Lacerda - MT - Brasil \\ pedroh.olives@gmail.com, \{renato.cani,joao.silva\}@plc.ifmt.edu.br
}

\begin{abstract}
Fake news are reports with false elements in their structure. They can interfere in important events, such as elections and preventive actions in pandemics. Thus, algorithms for classification and detection of these news promote interest in the scientific community. In this project, we develop a mathematical model based on modifications in a previous model for detecting fake news that considers structural features for classifying news as true or false. Besides, we implement a genetic algorithm for weighting the relevant factors. The results indicate a better efficacy in comparison to the model used as reference.
\end{abstract}

Resumo. Notícias falsas (fake news) são notícias com elementos inverídicos em sua estrutura e que podem interferir em eventos importantes, desde eleições a ações de combate a pandemias. Assim, algoritmos para classificação e detecção dessas notícias interessam à comunidade científica. Neste trabalho, desenvolvemos um modelo matemático baseado em modificações de um modelo anterior para detecção de fake news que considera componentes estruturais de notícias para sua classificação como verdadeira ou falsa. Além disso, implementamos um algoritmo genético para ponderação dos fatores de relevância. Resultados indicam uma melhor eficácia em comparação ao modelo utilizado como base.

\section{Introdução}

As fake news são notícias que apresentam informações inverídicas sobre determinado assunto. Essas notícias são disseminadas sobretudo nas redes sociais e nos sites da web. O fenômeno das fake news vem sendo especialmente discutido desde as eleições de 2016 nos EUA, quando a campanha de Donald Trump supostamente compartilhou nas redes sociais inúmeras notícias falsas sobre a sua oponente, Hillary Clinton [Persily 2017].

Considerando os problemas gerados a partir da disseminação das fake news, foi desenvolvido um modelo matemático inspirado em [Ferreira et al. 2020], tomado aqui como trabalho de referência, para classificar e detectar as notícias falsas publicadas em sites. O modelo deste trabalho estabelece sete critérios correspondentes a elementos estruturais de uma notícia, que foram selecionados usando como base o trabalho de referência [Ferreira, 2020], estes critérios são: $C_{1}$ ) Existência Autor; $C_{2}$ ) Título da notícia em caixa alta; $C_{3}$ ) Nota do Page Rank do site; $C_{4}$ ) Posição do site que veicula a notícia analisada no ranking do Google; $C_{5}$ ) Quantidade de notícias similares; $C_{6}$ ) Média do Page Rank das notícias similares; $C_{7}$ ) Média das posições dos sites que veiculam notícias similares.Para 
cada notícia, são extraídas informações que resultam em notas para cada um dos critérios. Em seguida, foi aplicado um algoritmo genético (AG) com o objetivo de encontrar o melhor balanceamento entre os pesos atribuídos a cada critério, a fim de identificar os fatores que mais impactam a classificação de notícias.

O objetivo deste trabalho é desenvolver um algoritmo genético que melhore a ponderação dos valores de cada critério, trazendo aprimoramento da acurácia da avaliação da veracidade das notícias. Ao todo, foi utilizado 100 notícias veiculadas em sites, sendo 50 delas para ajuste dos parâmetros do algoritmo genético e 50 delas para sua validação.

Considerando o estado da arte acerca do tema, observa-se trabalhos que abordam tanto aspectos da teoria sobre as fake news como da implementação de algoritmos para sua detecção, como [Marques et al. 2017]. Também é digno de nota o fato de que a definição do conceito de fake news é debatida há longa data, por profissionais de diferentes áreas do conhecimento, como citam Delmazo e Valente (2018). Constatando, assim, a atualidade e a relevância do tema em questão, bem como a existência de campo aberto para aprimoramentos na aplicação de algoritmos genéticos para classificação de fake news na Web.

\section{Materiais e Métodos}

\subsection{Modelo Matemático}

Nesta pesquisa, promovemos modificações no modelo matemático para classificação de fake news apresentado por [Ferreira et al. 2020] visando uma melhor taxa de acertos na identificação das notícias. Assim, a Equação 1 descreve a equação governante, que calcula a nota de uma notícia para classificação, em que $N$ é o número de critérios avaliados, $C_{i}$ é o valor associado a cada um deles e $P_{i}$ o peso ou fator de relevância de cada um.

$$
A_{i}=\sum_{i=1}^{N} P_{i} C_{i}-\Omega
$$

A primeira modificação no modelo consiste na inserção do fator $\Omega$ na equação governante. Esse fator penaliza a nota de notícias que possuam títulos similares veiculadas por outros sites através da existência de expressões pertencentes a um dicionário com termos comumente encontrados nos títulos de fake news. O valor de $\Omega$ é 0,1 como punição de $10 \%$ da nota máxima que a notícia pode receber, quando são desmentidas em outros sites e 0 em caso contrário.

As restrições do modelo são apresentadas pela Equação 2, que garante que a soma dos pesos atribuídos a cada critério resulte em 1, e pela Equação 3, que exige que as notas de cada critério sejam valores reais entre 0 e 1, inclusive. Isso assegura que, na Equação 1, a nota de um notícia mantenha-se no mesmo domínio. Logo, notícias com notas maiores do que 0,6 (ou 60\%) são classificadas como verdadeiras; em caso contrário, como falsas, de acordo com o [Ferreira et al. 2020].

$$
\sum_{i=1}^{N} P_{i}=1
$$




$$
C_{i} \in[0,1] \forall i \leq N
$$

Outra modificação realizada no modelo referência diz respeito aos critérios $C_{4}$ e $C_{7}$, nos quais inserimos faixas de avaliação que valorizam as notícias com melhores posições. Assim, ordena-se o ranking das notícias contidas na base. Usamos faixas em razão de melhorar na classificação já que antes as noticias Similares e as medias das noticias do Ranking eram divididas em um bilhão de noticias, e isso resultava em um número muito pequeno, que atrapalhava em classificar as notícias como True ou Fake. A Equação 4 expressa o critério $C_{4}$, na qual $F a_{i}$ representa um determinado numero de faixa escolhido, de acordo com a quantidade de notícias analisadas e $\alpha$ compreende o valor da razão entre 1 e o número de faixas. $\mathrm{O}$ critério $C_{7}$ foi modificado de modo análogo. Os demais critérios não foram alterados, como se lê em [Ferreira et al. 2020].

$$
C_{4}=1-F a_{i} \alpha+\alpha
$$

\subsection{Algoritmo Genético}

No trabalho referência, a relevância de todos os critérios é dada pelo fator 0,142 . Utilizamos, assim, um AG para variação desses pesos, visando obter uma melhor classificação, bem como identificar os critérios mais influentes. Os AG's são algoritmos de inteligência computacional inspirados na teoria da evolução e são facilmente aplicáveis a problemas de otimização, requerendo apenas a modelagem de indivíduos como exemplos de soluções e de uma função de aptidão a mensurar a qualidade dos indivíduos [Holland John 1975].

Em nosso trabalho, formulamos um indivíduo como sendo um vetor de 7 posições, no qual cada valor corresponde à relevância de um dos critérios. As restrições impostas pelas Equações 2 e 3 devem ser respeitadas por todos os indivíduos.

Para avaliar os indivíduos, foram utilizadas 50 notícias para ajuste de parâmetros, das quais 25 eram falsas e 25 verdadeiras. Nesse sentido, propõe-se como função aptidão a Equação 5, que visa a maximizar a diferença entre as notas obtidas pelas $M=25$ notícias verdadeiras e as $Q=25$ notícias falsas. Os parâmetros do algoritmo genético utilizados foram 100 indivíduos submetidos a 100 gerações com taxa de cruzamento de $50 \%$ e de mutação de $5 \%$.

$$
\max \left(\sum_{i=1}^{M} P_{i} C_{i}-\sum_{j=1}^{Q} P_{j} C_{j}\right)
$$

O extrator de valores dos critérios a partir da $U R L$ da notícia, a busca por notícias similares e o algoritmo genético foram implementados na linguagem de programação Python, com auxílio de bibliotecas (urllib.request, requests, search, difflib, urlparse, sys, json).

\section{Resultados e Discussão}

Após a execução do algoritmo genético aplicado ao modelo modificado proposto, utilizamos outras 50 notícias para validação dos resultados e comparamos ao trabalho referência. 
Nesse sentido, a Figura 1(a) apresenta os pesos encontrados pelo melhor indivíduo identificado pelo AG; já a Figura 1(b) mostra a comparação (em valores percentuais) das taxas de acerto na classificação obtidas pelos dois trabalhos.

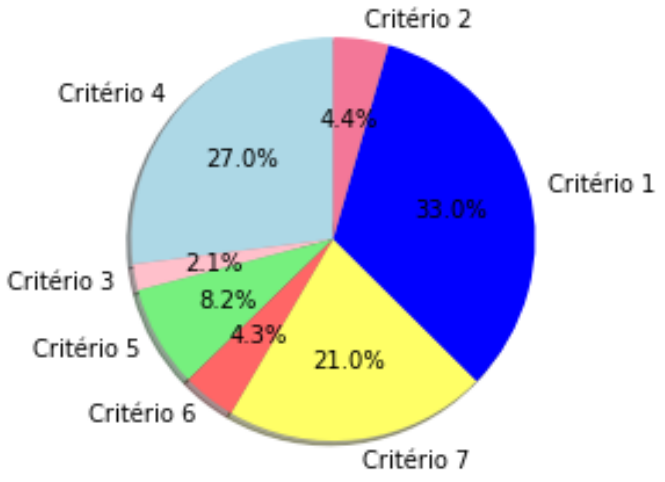

(a) Pesos encontrados pelo indivíduo

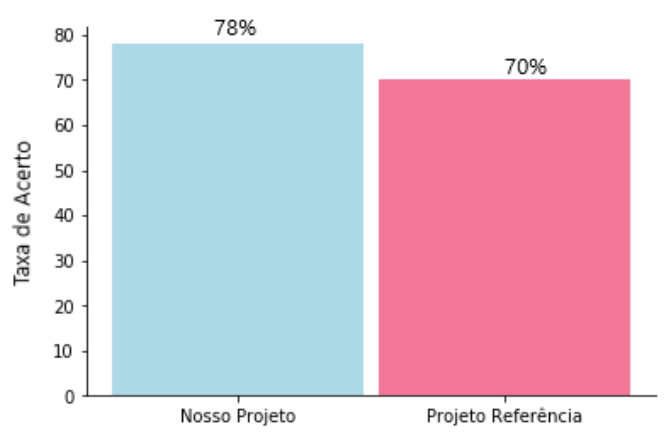

(b) Comparação entre as classificações

Figura 1. Principais resultados encontrados pelo Projeto

Na Figura 1(a), é possível observar que, para a base de dados testada, a existência de autor, posição do site que veicula a notícia no ranking e o ranking dos sites de notícias similares são os critérios com maior relevância na classificação das fake news. Além disso, na Figura 1(b), nota-se que as modificações apresentadas pela proposta a tornaram mais eficazes que o modelo referência, aumentando a taxa de acertos na classificação para $78 \%$ comparados com $70 \%$ alcançados pelo modelo original.

\section{Considerações Finais}

Neste trabalho, desenvolvemos um algoritmo genético para ajuste de parâmetros de um modelo matemático modificado para classificação de fake news na Web. A abordagem apresentada foi eficiente, visto que obteve taxa de acerto maior que a taxa alcançada pelo trabalho antes da modificação. Trabalhos futuros consistem na coleta de um maior número de notícias da Web e na utilização de outras técnicas de inteligência computacional.

\section{Referências}

Ferreira, A. L. N., Nascimento, D. G., Basílio, S. A. C., and SILVA, J. G. R. (2020). Um modelo matemático para classificação de fake news na web. Anais do LII Simpósio Brasileiro de Pesquisa Operacional.

Holland John, H. (1975). Adaptation in natural and artificial systems. Ann Arbor: University of Michigan Press.

Marques, T., da Silva Neto, O., and Diniz, D. (2017). Algoritmo genético aplicado ao problema de alocação/localização de facilidades. In Anais do IV Encontro Nacional de Computação dos Institutos Federais, pages 49-52. SBC.

Persily, N. (2017). The 2016 us election: Can democracy survive the internet? Journal of democracy, 28(2):63-76. 